\title{
Intrinsic, multiplexable sensors for electric field strength using structural slow light in phase-shifted fibre Bragg gratings
}

\author{
Philip Orr* and Paweł Niewczas \\ Institute for Energy and Environment, University of Strathclyde, Glasgow G1 1XW, UK
}

\begin{abstract}
In this paper we demonstrate through simulation the potential for phase-shifted fibre Bragg gratings incorporating structural slow light to enable intrinsic reflection-mode point sensors for electric field or voltage. It is shown that lo-bi FBGs incorporating multiple phase shifts yield large enhancements in group index (group delay) at resonance, thus amplifying and localizing time-dependent non-reciprocal effects. A relative, multiplexable measurement of electric field by comparison of the phase unbalance between linear modes on and off resonance is proposed, yielding static resolutions of $24 \mathrm{~V}$ and $18 \mathrm{mV}$ respectively in unpoled (dc Kerr effect) and poled (Pockels effect) fibres.
\end{abstract}

Keywords: slow light, voltage, electric field, fibre Bragg gratings, birefringence

\section{INTRODUCTION}

Recently, there has been a growing interest in the production and applications of structural slow light in uniform and phase-shifted fibre Bragg gratings (FBGs $)^{1-3}$. As opposed to alternative methods for the production of slow light ${ }^{4}$, resonances at the edges of an FBG bandgap offer a passive and relatively simple technique for the production of structural slow light in concentrated regions of the fibre and spectrum. In this case, the term "slow light" describes the effective increase in group delay and group index that is a result of the multiple reflections undergone at resonant wavelengths within the structure. Using such structures, it may be possible to greatly increase the sensitivity of gratingbased transducers to a variety of measurands. However, although the enhancements can be very large, the wavelength range over which the effect may be utilized is usually extremely narrow. This, and the requirement for operation in transmission, limits the integration of slow light grating sensors with conventional optical fibre sensor networks and interrogation topologies.

Measurement of voltage or electric field strength using optical fibre has thus far been facilitated by the Pockels effect (in poled fibre ${ }^{5}$ or bulk glass ${ }^{6}$ ), infiltration by liquid crystals ${ }^{7}$, or by using intermediate extrinsic materials (piezoelectricity ${ }^{8}$ or electrostriction ${ }^{9}$ ). Optical measurement of voltage is highly desirable in the electric power industry for diagnostic and protection purposes due to its inherent isolated and passive nature, and its ability to multiplex sensing points situated at diverse locations on the network ${ }^{10}$. This will become increasingly important in the near future with the introduction of distributed energy storage and generation. FBGs have been utilized to measure voltage-induced strain via a piezoelectric coating, and this has been successfully applied to multiplexed voltage measurement in subsea motors ${ }^{8}$. Although an intrinsic fibre point sensor for electric field strength may be more suitable for use in adverse environmental conditions, at high frequencies, or over a large voltage range, it remains undeveloped primarily due to the weakness of electro-optic effects in conventional fibre point sensors.

In this paper we propose an intrinsic fibre point sensor for electric field strength - and hence voltage - that utilizes structural slow light in reflection to overcome the weakness of the electro-optic (dc) Kerr effect or the linear electro-optic effect within FBGs. The resulting transducers may be serially-multiplexed and interrogated in reflection mode using an associated interrogation technique described herein, and may incorporate the simultaneous measurement of temperature by conventional methods.

\section{PRINCIPLE OF SLOW-LIGHT VOLTAGE TRANSDUCER}

It has been shown previously that enhanced, tailored group delay in reflection can be achieved at the Bragg wavelength by the superposition of resonances induced by $\pi$ phase shifts in the index modulation, and that simulated group

*philip.orr@ strath.ac.uk; phone +44 (0)141 548 4841; fax +44 (0)141 548 4872; www.instee.strath.ac.uk 
delays can be achieved in practice in low-birefringence gratings ${ }^{11}$. It is of interest whether the enhancement achievable in such structures will permit the in-fibre measurement of electric field, E. Within a grating, the field will produce a linear birefringence along the direction of the field lines via the nominally weak electro-optic Kerr effect in standard silica fibre,

$$
\Delta \mathrm{n}_{\mathrm{E} \_\mathrm{K}}=\lambda K E^{2}=\lambda K V^{2} / l^{2}
$$

or via the linear electro-optic (Pockels) effect in poled silica fibres,

$$
\Delta \mathrm{n}_{\mathrm{E} \_\mathrm{P}}=\mathrm{n}^{3} \mathrm{r} E / 2=\mathrm{n}^{3} \mathrm{r} V / 21 .
$$

In (1), $K$ is the Kerr coefficient ${ }^{12}\left(5.3 \times 10^{-16} \mathrm{~m} \cdot \mathrm{V}^{-2}\right.$ at $\left.23^{\circ} \mathrm{C}\right), V$ is voltage between electrodes, $\lambda$ is optical wavelength, and $l$ is the gap between electrodes within which the fibre sensor is situated. In (2), $r$ is the Pockels coefficient and $n$ is refractive index. Figure 1a illustrates the logarithmically-spaced phase-shifts of the optimal sensor structure ${ }^{2}$ (where overlap of resonances is maximized), and the geometry of the voltage transducer. The graph in Figure $1 \mathrm{~b}$ was produced using the $4 \times 4$ state matrix analysis method ${ }^{13}$ in order to demonstrate the enhancement to effective path length at the resonant (Bragg) wavelength within the grating spectrum. In this structure, five phase shifts are used, yielding an enhancement factor (ratio of peak delay to off-resonance delay) of approximately 670. Note that the reflectivity of the grating over this region is close to $100 \%$.

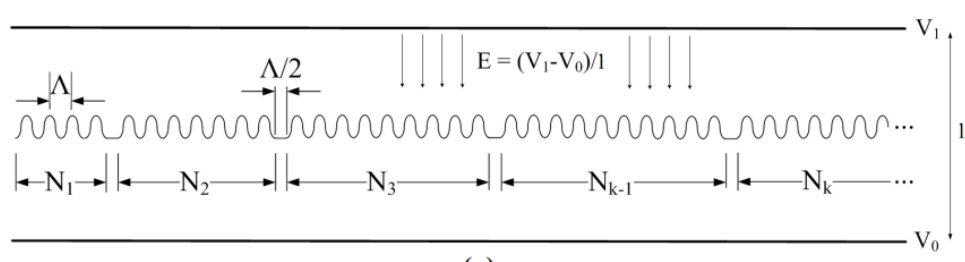

(a)

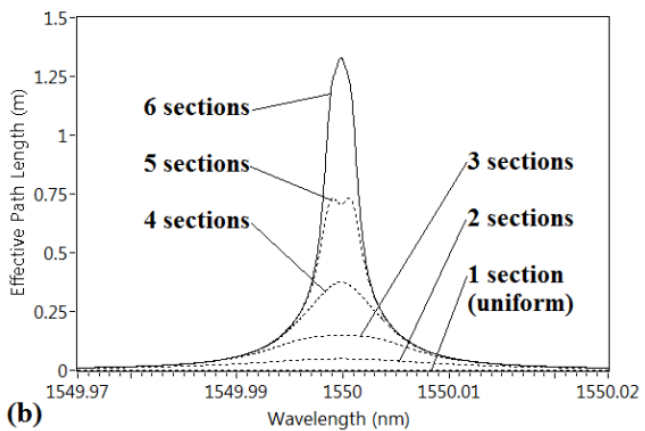

(b)

Figure 1. (a) Multi-phase-shift grating structure (substructure ratio 3/7/8.75/10/11/20, total length $31 \mathrm{~mm}$ ) for enhanced group delay in reflection, configured for voltage measurement. (b) The wavelength-dependent enhancement of effective path length in the same structure. The peak enhanced effective path length is referred to in Section 3 as $d_{\text {eff }}$.

It is clear that the significant enhancement of effective path length $d_{\text {eff }}$ at a narrowband region within the Bragg spectrum will allow for the localized enhancement of the optical path difference $\mathrm{d}_{\mathrm{eff}} \Delta \mathrm{n}_{\mathrm{E}}$ arising from an incident electric field. Further, due to the wavelength-selectivity of the enhancement, there exists the possibility for a relative measurement of birefringence-inducing fields, as is proposed in the next section.

\section{SENSOR INTERROGATION}

Unlike magnetic field measurement, where the construction of reciprocal paths for differential phase measurement is straightforward, measurement of an induced linear birefringence does not invite the construction of reciprocal paths if multiplexing is desired. One solution is to make instead a relative measurement of the electric field induced birefringence permitted by the wavelength-dependence of the transducer sensitivity shown in Figure 1b. This may be achieved by comparison of the phase unbalance $\Delta \phi$ at an off-resonance region of the FBG spectrum with that at the resonance superposition.

With reference to Figure 2, a tunable narrowband source may be incident upon the sensor gratings via a balanced MachZehnder interferometer and polarization beam splitter (with inputs both aligned to slow axis), such that light travelling to sensors and back on the PM slow and fast axes are brought to interference at the $3 \times 3$ coupler where their phase difference may be determined robustly ${ }^{14}$. As the scanning source moves between gratings, and between regions of minimum and maximum resonance (effective path length) within individual gratings, the phase difference incurred between orthogonal linear modes at off-resonance wavelengths within grating spectra is given by

$$
\Delta \phi_{\mathrm{OFF}}=(2 \pi / \lambda) \cdot 2 d_{P M} \cdot \Delta n_{\mathrm{PM}}
$$


where $d_{P M}$ is the distance from the PBS to FBG, and $\Delta n_{\mathrm{PM}}$ is the PM fibre birefringence $\left(0.3 \times 10^{-3}\right.$ for $5 \mathrm{~mm}$ beat length). At the resonant enhancement, the effective path length $d_{\text {eff }}$ is greatly increased as shown in Figure 1. Under conditions of incident electric field, the phase difference at resonance will thus be

$$
\Delta \phi_{\mathrm{ON}}=\Delta \phi_{\mathrm{OFF}}+(2 \pi / \lambda) \cdot \mathrm{d}_{\mathrm{eff}} \Delta \mathrm{n}_{\mathrm{E}}
$$

where $\Delta \mathrm{n}_{\mathrm{E}}$ is the additional birefringence induced by the aligned transverse electric field, which affects $\Delta \phi_{\mathrm{OFF}}$ by a negligible amount over the length of the FBG.

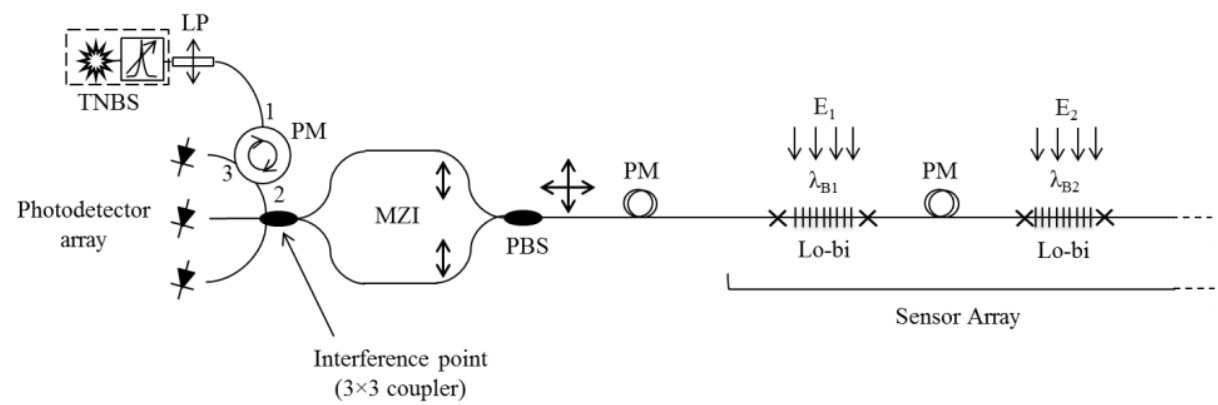

Figure 2. Reflection-mode interrogation scheme for measurement of electric field strength and temperature within serially multiplexed slow-light FBGs. TNLS is tunable narrow linewidth source (tunable laser or filtered broadband source), LP is linear polarizer, PBS is polarization beam splitter/combiner, LB is low-birefringence, PM is polarizationmaintaining (hi-bi).

In practice, the scheme could use an intensity threshold to begin and end phase calculation and tracking. After the grating has been scanned to map the phase difference across its spectrum, the difference $\Delta \phi_{\mathrm{ON}}-\Delta \phi_{\mathrm{OFF}}$ may be found, yielding $(2 \pi / \lambda) \mathrm{d}_{\mathrm{eff}} \Delta \mathrm{n}_{\mathrm{E}}$. By characterization of the grating during fabrication, $\mathrm{d}_{\mathrm{eff}}$, which is a gauge of the sensitivity, will be predetermined. Following calculation of $\Delta n_{\mathrm{E}}$, the electric field magnitude may be calculated using either (1) or (2) depending on the type of fibre used.

A simulation of the grating's response to electric field is shown in Figure 3 for both conventional and poled fibres ${ }^{15}$, based on the example sensor structure of Figure 1. With respect to our laboratory noise floor of $50 \mu \mathrm{rad} / \mathrm{Hz}^{1 / 2}$, measurement noise floors of $3.4 \mathrm{~V} / \mathrm{Hz}^{1 / 2}$ (un-poled) and $1.8 \times 10^{-3} \mathrm{~V} / \mathrm{Hz}^{1 / 2}$ (poled) can be estimated for an electrode gap of $150 \mu \mathrm{m}$. At $100 \mathrm{~Hz}$, static resolutions of $34 \mathrm{~V}$ or $18 \mathrm{mV}$ respectively may be expected - alternatively the wavelength may be dithered at the enhancement to amplitude modulate the differential phase unbalance onto a carrier for improved resolution. It should also be noted that the interrogation scheme described here is an illustrative one, and that alternative schemes will be compatible with this in-grating approach, such as Michie' ${ }^{5}$ which may offer improved resolution and simplicity at the expense of less robust phase demodulation.
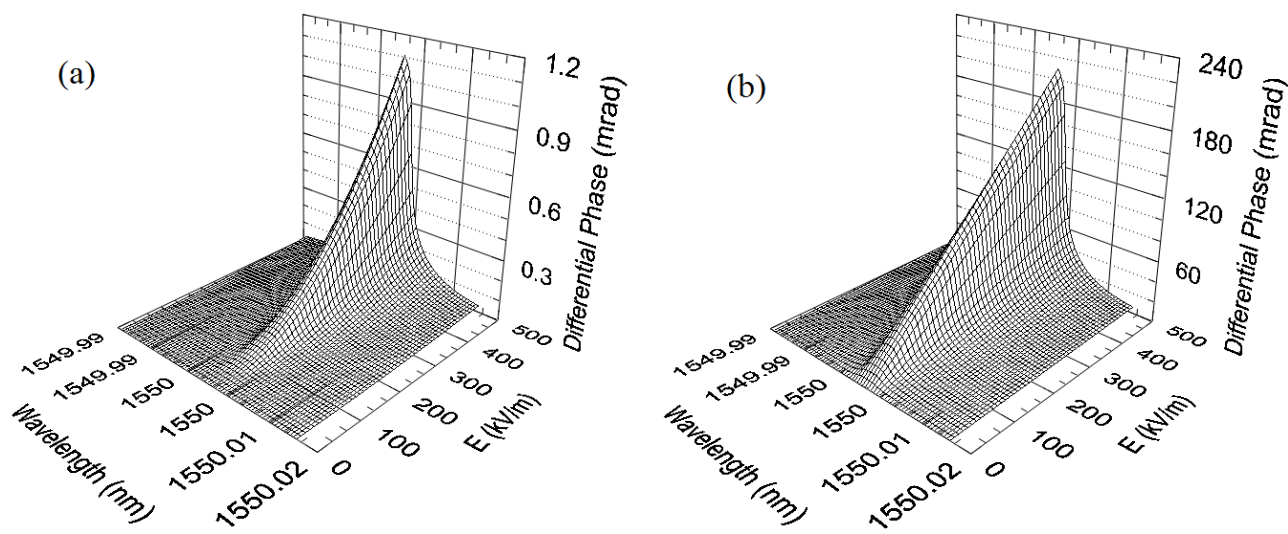

Figure 3. Simulated phase unbalance spectrum in low-birefringence multi-shift grating (design in Figure 1) subject to (a) the electro-optic Kerr effect (lo-bi silica fibre, no poling) and (b) the linear electro-optic effect $(\mathrm{r}=0.25 \mathrm{pm} / \mathrm{V})$ in poled silica fibre via an increasing transverse electric field strength. 
Note that in this scheme it is assumed that environmental perturbations in $\Delta \mathrm{n}_{\mathrm{PM}}$ change much more slowly than the time to scan from off-resonance to on-resonance, and therefore will produce only a common-mode offset that does not affect the differential measurement. Wavelength multiplexing capability is retained since the differential measurement will be unaffected by other gratings on the same fibre. Finally, for simultaneous measurement of temperature or longitudinal strain, the Bragg wavelength shift - which will not corrupt the superposition of resonances ${ }^{2}$ - may be detected in parallel in the conventional manner.

\section{CONCLUSIONS}

In this paper we have illustrated the potential for fibre Bragg gratings incorporating structural slow light to overcome the weak electro-optic Kerr effect in standard silica fibre, or to enhance and localize the linear electro-optic effect in poled fibres for point sensing purposes. Through simulation it was shown that lo-bi FBGs incorporating multiple phase shifts at logarithmically-spaced locations are able to yield a factor of 670 enhancement in group index (group delay) at resonance. Thus, it is proposed that this enhancement may be used to amplify and localize time-dependent non-reciprocal effects, leading to intrinsic sensors for electric field (hence voltage). In order to interrogate the structures, we propose a relative measurement of electric field by comparison of the phase unbalance between linear modes on and off resonance, yielding static resolutions of $24 \mathrm{~V}$ and $18 \mathrm{mV}$ respectively in unpoled and poled fibres. The sensors may be wavelength multiplexed and their Bragg shift under heating or strain can be extracted simultaneously. It is clear that the proposed transducers may also enable miniature voltage-tuned fibre delays and isolators.

\section{REFERENCES}

[1] Wen, H., Terrel, M., Fan, S., and Digonnet, M., "Sensing With Slow Light in Fiber Bragg Gratings,” IEEE Sensors J. 12(1), 156-163 (2012)

[2] Orr, P., Niewczas, P., Stevenson, M., and Canning, J., "Compound Phase Shifted Fibre Bragg Structures as Intrinsic Magnetic Field Sensors,” J. Lightwave Tech. 28(18), 2667-2673 (2010)

[3] Qian, K., Zhan, L., Li, H., Hu, X., Peng, J., Zhang, L., and Xia, Y., “Tunable delay slow-light in an active fiber Bragg grating," Opt. Express 17, 22217-22222 (2009)

[4] Slepkov, A. D., Bhagwat, A. R., Venkataraman, V., Londero, P., and Gaeta, A. L., "Generation of large alkali vapor densities inside bare hollow-core photonic band-gap fibers," Opt. Express 16(23), 18976-18983 (2008)

[5] Michie, A., Bassett, I. M., Haywood, J. H., and Ingram, J., "Electric field and voltage sensing at $50 \mathrm{~Hz}$ using a thermally poled silica optical fibre," Meas. Sci. Tech. 18, 3219-3222 (2007)

[6] Pan, F., Xiao, X., Xu, Y., and Ren, S., “An Optical AC Voltage Sensor Based on the Transverse Pockels Effect," Sensors (Basel) 11(7), 6593-6602 (2011)

[7] Wolinski, T. R., Ertman, S., Lesiak, P., Domanski, A. W., Czapla, A., Dabrowski, R., Nowinowski-Kruszelnicki, E., and Wojcik, J., "Photonic Liquid Crystal Fibers: A New Challenge for Fiber Optics and Liquid Crystals Photonics," Opto-electronics Review, 14(4), 329-334 (2006)

[8] Dziuda, L., Niewczas, P., Fusiek, G., and McDonald, J. R., "Hybrid Fiber-Optic Voltage Sensor for Remote Monitoring of Electrical submersible Pump Motors", Opt. Eng. 44(6) (2005)

[9] Bonhert, K., Gabus, P., Kostovic, J., and Brändle, H., "Optical fiber sensors for the electric power industry," Optics in Lasers and Engineering 43, 511-526 (2005)

[10] Parker, D.M. and McCollough, N.D., "Medium-voltage sensors for the smart grid: Lessons learned," IEEE Power and Energy Society General Meeting, 1-7, 24-29 July 2011

[11] Orr, P., Niewczas, P., Stevenson, M., and Canning, J., "Automatable Fabrication of Dispersion-Tailored Bragg Gratings for Tunable Narrowband Delays," Elec. Lett., 46(18), 1283-1284 (2010)

[12] Farries, M. C. and Rogers, A. J., "Temperature dependence of the Kerr effect in a silica optical fibre," Elec. Lett. 19(21), 890-891 (1983)

[13] Sakaguchi, S. and Sugimoto, N., "Transmission Properties of Multilayer Films Composed of Magneto-Optical and Dielectric Materials," J. Lightwave Tech. 17(6), 1087-1092 (1999)

[14] Todd, M. D., Seaver, M., and Bucholtz, F., "Improved, operationally-passive interferometric demodulation method using $3 \times 3$ coupler," Elec. Lett. 38(15), 784-786 (2002)

[15] Srinivasan, B. and Jain, R. K., "First Demonstration of Thermally Poled Electrooptically Tunable Fiber Bragg Gratings,” IEEE Phot. Tech. Lett. 12(2), 170-172 (2000) 